\title{
Results of Hygiene Education of Kitchen Stove Knob and Water Faucet by using ATP Inspection
}

\author{
By Naomi Katayama, Shoko Kondo, Akemi Ito, Mayumi Hirabayashi, Yui Nakayama, \\ Ayari Naka, Natuki Sasaki, Moe Inuzuka \& Takashi Tamura \\ Nagoya Women's University
}

Abstract- In Japan, many food poisoning cases occur every year form the rainy season to summer. After all, restaurants account for about $50 \%$ of all food poisoning cases. Still they also occur at school meals $(6.4 \%)$ and hospitals $(0.6 \%)$, where hygiene management should strictly controlled in the Ministry of Health, Labor, and Welfare announced (2019). Therefore, from the viewpoint of food poisoning prevention, this study focused on the stove knob and water faucet, which are often touched by the cook's fingers in the kitchen and may cause secondary contamination. The effect of hygiene education investigated by measuring invisible microbial load as visible ATP value using ATP wiping test. The 12 stove knob and 11 water faucet inspected before and after hygiene education by using the ATP wiping test. Before hygiene education, the average value of the ATP values measured after washing before and after washing. To the surprise, after washing, the ATP value did not drop below 1000 for Stove knob and Water faucet. Almost the same ATP value as before cleaning.

Keywords: gender; ATP wiping test, stove knob, water faucet, Hygiene education, double wash.

GJMR-K Classification: NLMC Code: QT 180

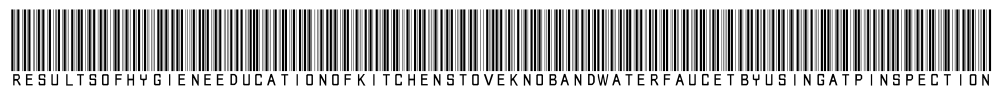

Strictly as per the compliance and regulations of:

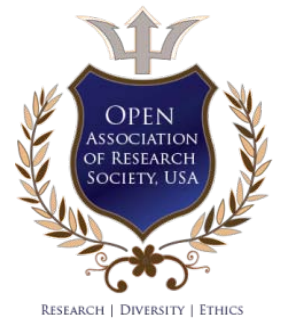

(c) 2020. Naomi Katayama, Shoko Kondo, Akemi Ito, Mayumi Hirabayashi, Yui Nakayama, Ayari Naka, Natuki Sasaki, Moe Inuzuka \& Takashi Tamura. This is a research/review paper, distributed under the terms of the Creative Commons Attribution-Noncommercial 3.0 Unported License http://creativecommons.org/licenses/by-nc/3.0/), permitting all non-commercial use, distribution, and reproduction in any medium, provided the original work is properly cited. 


\title{
Results of Hygiene Education of Kitchen Stove Knob and Water Faucet by using ATP Inspection
}

\author{
Naomi Katayama ${ }^{\alpha}$, Shoko Kondo ${ }^{\circ}$, Akemi Ito ${ }^{\circ}$, Mayumi Hirabayashi ${ }^{\omega}$, Yui Nakayama ${ }^{\ddagger}$, Ayari Naka ${ }^{\S}$, \\ Natuki Sasaki ${ }^{x}$, Moe Inuzuka ${ }^{\vee} \&$ Takashi Tamura $^{\ominus}$
}

\begin{abstract}
In Japan, many food poisoning cases occur every year form the rainy season to summer. After all, restaurants account for about $50 \%$ of all food poisoning cases. Still they also occur at school meals $(6.4 \%)$ and hospitals $(0.6 \%)$, where hygiene management should strictly controlled in the Ministry of Health, Labor, and Welfare announced (2019). Therefore, from the viewpoint of food poisoning prevention, this study focused on the stove knob and water faucet, which are often touched by the cook's fingers in the kitchen and may cause secondary contamination. The effect of hygiene education investigated by measuring invisible microbial load as visible ATP value using ATP wiping test. The 12 stove knob and 11 water faucet inspected before and after hygiene education by using the ATP wiping test. Before hygiene education, the average value of the ATP values measured after washing before and after washing. To the surprise, after washing, the ATP value did not drop below 1000 for Stove knob and Water faucet. Almost the same ATP value as before cleaning. It turned out that the number of microorganisms did not decrease even if the cooks cleaned themselves. Then, the inspector wash the stove knob and water faucet firmly with detergent and sponge, wipe clean with a clean cloth three times or more. The inspector taught the cooks to repeat this process twice. The cook tries to do it as same as the inspector is doing. Then, the cook tries to do it next cooking. After hygiene education, the average value of the ATP values was significant low. Unfortunately, after washing, the ATP value did not drop below 100 for the Stove knob and Water faucet. Even after the hygiene education, the ATP value did not fall below 100 , an indication that more rigorous hygiene education is necessary.
\end{abstract}

Keywords: gender; ATP wiping test, stove knob, water faucet, Hygiene education, double wash.

\footnotetext{
Corresponding Author a: Naomi Katayama, Nagoya Women's University, Nagoya City, Japan, Graduate School of Nagoya Women's University, Nagoya City, Japan, Department of Otorhinolaryngology, Nagoya University Graduate School of Medicine, Nagoya, Japan.

e-mail:naomik@nagoya-wu.ac.jp

Author $\sigma$ : Watanabe Hospital, Mihama town, Noma, Aichi, Japan.

Author $\rho \omega$ : Graduate School of Nagoya Women's University, Nagoya City, Japan.

Author $¥$ : Nagoya cooking School, Nagoya, Aichi, Japan.

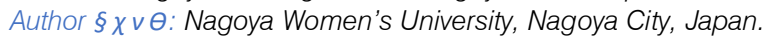

\section{INTRODUCTION}

( utbreaks of food poisoning often involve cooking and providing food together, for example, school lunches, employee cafeterias, and inns with meals. Therefore, to the Japanese Ministry of Health, Labor, and Welfare, it is necessary to strictly management of hygiene. HACCP (Hazard Analysis Critical Control Point) is a base method of the hygiene management. NASA used this system to prevent Hazards related to food handling in advance. This HACCP has 12 items, and the $9^{\text {th }}$ measurement method (monitoring) setting ${ }^{1)}$. Currently, the ATP wipe test used to control microorganisms in hospitals and food companies ${ }^{2,3,4)}$. Food poisoning in elderly facilities is likely to cause death; this is a reason why hygiene education for the staff is essential(5). Since microorganisms are invisible, it is possible to count the number of bacteria as the number of ATP by using the ATP wiping test. This test is very useful for health education. The cook's fingers touch many places in the kitchen. And, depending on the place, there are places where the fingers of multiple cooks touched many times. For example the place, a stove knob or a water faucet. Therefore, it is necessary to repeat the hygiene education of the cooks and give a careful explanation about the places where the cook's fingers touch. The purpose of this study, the effect of hygiene education for cooks by conducting an ATP wiping test on the stove knob and water faucet installed in the kitchen.

\section{il. Materials and Methods}

\section{a) Stove knob and Water faucet}

The 12 stoves knob and 11 water faucet, prepared in the kitchen were wiped clean before the start of cooking. The stove knob ignites as it releases gas when pressed. So, every time the stove lit, the cook's finger will touch the stove knob. Similarly, the water faucet is the place where the cook's finger touches each time to uses water. Five cooktops have two stove knob and two water faucet, and one cooktop has two stove knob and one water faucet. 


\section{b) ATP inspection procedure}

Twelve cooks worked in groups of two to cook on six cooktops. Before the education of hygiene, the work start time depends on the working conditions of the cooks, but the inspector always performed an ATP inspection before using the 12 stove knob and 11 water faucet. Then, each cook finished the work, washed the 12 stove knob and 11 water faucet by himself, and they inspected the ATP inspection by inspector again. The value of ATP recorded. In the same way, after the education of hygiene, the work start time depends on the working conditions of the cooks. Still the inspector always performed an ATP inspection before using the 12 stove knob and 11 water faucet. Then, each cook finished the work, washed the 12 stove knob and 11 water faucet by himself, and they inspected the ATP inspection by inspector again. The value of ATP recorded.

\section{ili. Hygiene Education Procedure}

\section{a) Cleaning instruction}

While showing the ATP result before the hygiene education to the cook, ATP inspector the stove knob and the water faucet firmly with detergent and sponge, wipe clean with a clean cloth three times or more. Then, the inspector washes again the stove knob and water faucet carefully with detergent and sponge, wipe clean with a clean cloth three times or more. The cook tries to

Table 1: ATP test result of the stove knob before cleaning instruction

\begin{tabular}{lcc}
\hline \hline & \multicolumn{2}{c}{$\begin{array}{c}\text { before cleaning } \\
\text { instruction }\end{array}$} \\
\cline { 2 - 3 } Place & before & after \\
\hline stove 1 & 2730 & 285 \\
stove 2 & 1178 & 356 \\
stove 3 & 21 & 409 \\
stove 4 & 1593 & 432 \\
stove 5 & 6279 & 478 \\
stove 6 & 1254 & 493 \\
stove 7 & 388 & 906 \\
stove 8 & 634 & 1013 \\
stove 9 & 496 & 1131 \\
stove 1 0 & 1672 & 1644 \\
stove 1 1 & 8837 & 3677 \\
stove 1 2 & 774 & 4204 \\
\hline Average & 2154.67 & 1252.33 \\
Standard deviation & 2679.07 & 1322.4 \\
Median & 1216 & 699.5 \\
Maximum & 8837 & 4204 \\
Minimum & 21 & 285 \\
\hline \hline
\end{tabular}

b) After hygiene education: Stove knob and Water faucet

The results of ATP wiping tests on the stove knob and the water faucet after hygiene education shown in Table 3 and Table 4. It can see that the do it as same as the inspector is doing. Then, the cook tries to do it next cooking. After the educational, results of ATP recorded.

\section{b) Statistical processing}

The results obtained compared using statistical methods. The data to be compared was subjected to an $F$ test to determine whether to use a parametric test or nonparametric test. When there is no difference in the $F$ test, the presence or absence of a significant difference was confirmed using the student-t-test with or without a correspondence. If there was a difference in the F test, the presence or absence of a significant difference was confirmed using the Wilcoxon test with a pair or the Mann-Whitney test without correlation.

\section{Results}

\section{a) Before hygiene education: Stove knob and Water faucet}

The results of ATP wiping tests on the stove knob and the water faucet before hygiene education shown in Table 1 and Table 2. It can see that the average value of the ATP values measured after washing before and after washing and washing little bit lowers the ATP value. To the surprise, after cleaning, the ATP value did not drop below 1000 for Stove knob and Water faucet. Almost the same ATP value as before cleaning.

Table 2: ATP test result of water faucet before cleaning instruction

\begin{tabular}{lcc}
\hline \hline & \multicolumn{2}{c}{$\begin{array}{c}\text { before cleaning } \\
\text { instruction }\end{array}$} \\
\cline { 2 - 3 } Place & before & after \\
\hline water supply 1 & 574 & 428 \\
water supply 2 & 8848 & 531 \\
water supply 3 & 877 & 554 \\
water supply 4 & 6122 & 709 \\
water supply 5 & 2495 & 1639 \\
water supply 6 & 1850 & 3758 \\
water supply 7 & 54 & 4227 \\
water supply 8 & 2795 & 4442 \\
water supply 9 & 908 & 4922 \\
water supply 1 0 & 5499 & 6306 \\
water supply 1 1 & 28180 & 19893 \\
\hline Average & 5291.09 & 4309.91 \\
Standard deviation & 8071.87 & 5577.43 \\
Median & 2495 & 3758 \\
Maximum & 28180 & 19893 \\
Minimum & 54 & 428 \\
\hline \hline
\end{tabular}

average value of the ATP values measured after washing before and after washing and the ATP value significantly low. Unfortunately, after washing, the ATP value did not drop below 100 for the Stove knob and Water faucet. 
Table 3: ATP test result of the stove knob after cleaning instruction

\begin{tabular}{lcc}
\hline & \multicolumn{2}{c}{$\begin{array}{c}\text { after cleaning } \\
\text { instruction }\end{array}$} \\
\cline { 2 - 3 } Place & before & after \\
\hline stove 1 & 102 & 25 \\
stove 2 & 137 & 26 \\
stove 3 & 344 & 31 \\
stove 4 & 630 & 32 \\
stove 5 & 902 & 36 \\
stove 6 & 5643 & 59 \\
stove 7 & 2157 & 139 \\
stove 8 & 293 & 167 \\
stove 9 & 39 & 172 \\
stove 1 0 & 949 & 203 \\
stove 1 1 & 448 & 232 \\
stove 1 2 & 875 & 245 \\
\hline Average & 1043.25 & 113.917 \\
Standard deviation & 1559.37 & 87.4855 \\
Median & 539 & 99 \\
Maximum & 5643 & 245 \\
Minimum & 39 & 25 \\
\hline \hline
\end{tabular}

c) Statistical processing results

i. Comparison of ATP test values of Stove knob and Water faucet: before and after education

Before and after hygiene education, the results of the ATP wiping test on the stove knob and the water faucet statistically compared. The results shown in Tables 5 and 6 . The ATP wiping test values after hygiene
Table 4: ATP test result of water faucet after cleaning instruction

\begin{tabular}{lcc}
\hline \hline & \multicolumn{2}{c}{$\begin{array}{c}\text { after cleaning } \\
\text { instruction }\end{array}$} \\
\cline { 2 - 3 } Place & before & after \\
\hline water supply 1 & 153 & 1 \\
water supply 2 & 187 & 9 \\
water supply 3 & 1596 & 15 \\
water supply 4 & 91 & 15 \\
water supply 5 & 619 & 25 \\
water supply 6 & 183 & 110 \\
water supply 7 & 116 & 114 \\
water supply 8 & 3802 & 126 \\
water supply 9 & 54131 & 200 \\
water supply 1 0 & 712 & 315 \\
water supply 1 1 & 794 & 338 \\
\hline Average & 5671.27 & 115.273 \\
Standard deviation & 16109 & 122.242 \\
Median & 619 & 110 \\
Maximum & 54131 & 338 \\
Minimum & 91 & 1 \\
\hline \hline
\end{tabular}

education for the stove knobs and the water faucet were statistically significant difference. Although there was a statistically significant difference even before hygiene education, the ATP wiping test values for both were not less than 100 , so it can say that hygiene is still insufficient.

Table 5: Statistical comparison results : ATP test results of stove knob before andafter cleaning instruction

\begin{tabular}{lcccc}
\hline \hline & \multicolumn{2}{c}{ before cleaning instrucion } & \multicolumn{2}{c}{ after cleaning instruction } \\
\hline & before cooking & after cooking & before cooking & after cooking \\
\hline Average & 2154.7 & 1252.3 & 1043.3 & 113.9 \\
Standard deviation & 2679.1 & 1322.4 & 1559.4 & 87.5 \\
F test & $\mathrm{p}=0.011^{*}$ & $\mathrm{p}=0.0001^{* *}$ & \\
Student-t test & & \multicolumn{2}{c}{$\mathrm{p}=0.006^{* *}$} \\
Wilcoxon test & $\mathrm{p}=0.239$ & & \\
\hline \hline
\end{tabular}

$* \mathrm{P}<0.05,{ }^{* *} \mathrm{P}<0.01$

Table 6: Statistical comparison results : ATP test results of water faucet before and after cleaning instruction

\begin{tabular}{lcccc}
\hline \hline & \multicolumn{2}{c}{ before cleaning instrucion } & \multicolumn{2}{c}{ after cleaning instruction } \\
\cline { 2 - 5 } & before cooking & after cooking & before cooking & after cooking \\
\hline Average & 5291.1 & 4309.9 & 5671.3 & 115.3 \\
Standard deviation & 8071.9 & 5577.4 & 16109.0 & 122.2 \\
F test & $\mathrm{p}=0.118$ & $\mathrm{p}=0.0001^{* *}$ & \\
Student-t test & $\mathrm{p}=0.481$ & \multicolumn{2}{c}{$\mathrm{p}=0.003^{* *}$} \\
Wilcoxon test & & \multicolumn{3}{c}{} \\
\hline
\end{tabular}

$* \mathrm{P}<0.05,{ }^{* *} \mathrm{P}<0.01$ 


\section{Discussion}

The ATP test is a quick test because it can show the number of microorganisms by the ATP amount within one minute ${ }^{6,7)}$. Many hospitals have adopted this method to help maintain a hygienic environment ${ }^{8}$. In this study, the ATP wiping test used to inspect the stove knob and water faucet in the kitchen. The ATP wiping test used to show the number of microorganisms to the cooks, and the hygiene education conducted. The ATP value after washing the stove knob and water faucet before hygiene education showed almost the same ATP value as before cooking. The ATP value did not drop below 1000 for Stove knob and Water faucet. It is not change as ATP value as before cleaning. The microorganisms could not be removed by the cook's washing. However, after the hygiene education by the demonstration of the washing method by the inspector, hygienic cleaning with a statistically significant difference was achieved. Although there was a statistically significant difference even before hygiene education, the ATP wiping test values for both were not less than 100, so it can say that hygiene is still insufficient. It found that cooks need to regularly clean stove knobs and water faucet that is touched by multiple cooks even during cooking, and wipe with a clean cloth three or more times.

Although it has said that food poisoning is unlikely to occur during the cold season, Japan's Ministry of Health, Labor, and Welfare had revealed that noroviruses often occur in winter. Also, according to the announcement from the National Institute of Infectious, there are food poisoning $s$ caused by dysentery, cholera, typhoid, salmonella, and pathogenic Escherichia coli as import infectious diseases brought in from overseas. Food poisoning occurs when various factors such as foodstuffs, cooking utensils, fingers of cooks, and temperature and humidity of food storage overlap. To prevent this, such a cook needs to be highly aware of the hygiene management. Also, it is necessary to maintain hygiene management awareness by regularly measuring the ATP value using the AYP wiping test so that invisible microorganisms can see.

\section{Vi. Conclusions}

To prevent food poisoning, which may cause fatal accidents in some cases, we examined ways to improve instruction of hygiene education. The effect of hygiene education investigated by measuring invisible microbial load as visible ATP value using ATP wiping test. The 12 stove knob and 11 water faucet inspected before and after hygiene education by using the ATP wiping test. Before hygiene education, the average value of the ATP values measured after washing before and after washing. To the surprise, after washing, the ATP value did not drop below 1000 for Stove knob and Water faucet. There was no statistically significant difference between the ATP values measured at the start of cooking and after washing after completion. The ATP value is almost the same as before cleaning. It turned out that the number of microorganisms did not decrease even if the cooks cleaned themselves. Then, the inspector washes the stove knob and water faucet firmly with detergent and sponge, wipe clean with a clean cloth three times or more. The inspector taught the cooks to repeat this process twice. The cook tries to do it as same as the inspector is doing. Then, the cook tries to do it next cooking. After hygiene education, the average value of the ATP values was significant lowers. Unfortunately, after washing, the ATP value did not drop below 100 for the Stove knob and Water faucet. However, the ATP value was statistically significant. Even after the hygiene education, the ATP value did not fall below 100, an indication that more rigorous hygiene education is necessary.

\section{ACKNOWLEDGEMENTS}

We would like to thank all the cooks who participated in this experiment. Also, we would like to thank the inspectors who also performed the ATP inspection.

\section{References Références Referencias}

1. Osimani A, Garofalo C, Clementi F, Tavoletti S, Aquilanti L. Bioluminescence ATP monitoring for the routine assessment of food contact surface cleanliness in a university canteen. (2014). Int J Environ Res Public Health 17; 11(10): 10824-10837.

2. Nante N, Ceriale E, Messina G, Lenzi D, Manzi P. Effectiveness of ATP bioluminescence to assess hospital cleaning: a review. (2017) J Prev. Med. Hyg. 58(2): E177-E183.

3. Amodio E, Dubi C. Use of ATP bioluminescence for assessing $h$ eclealiness of hospital surfaces: a review of the published literature (1990-2012). (2014) J infect Public Health 7(2): 92-98.

4. Aycieck H, Oquz U. Karci K. Comparison of results of ATP bioluminescence and traditional ygiene swabbing methods for the deteminaton of surface cleanliness at a hospital kitchen. (2006). Int J Hyg Environ Health. 209(2): 203-206.

5. Lee JH (2018) An investigation of Factors that influence Hygiene Practices at a small Day Care Center. (2018). J Food Prot. 81(1): 158-164.

6. Stanley PE. A review of bioluminescent STP techniques in papid microbiology. (1989) J Biolumin Chemilumin 4(1): 375-380.

7. Stannard CJ, Gibbs PA. Rapid microbiology: application $s$ of bioluminescence in the food industry - a review. (1986) J Biolumin Chemilumin 1(1): 3-10.

8. Griffith CJ, Coooper RA, Gilmore J, Davies C, Lweis $M$. An evaluation of hospital cleaning refimes and standards. (2000) J Hosp Infect. 45(1): 19-28. 